\title{
Surgical Treatment of Moyamoya Disease in Adults: Combined Direct and Indirect vs. Indirect Bypass Surgery
}

\author{
Dal-Soo KIM, ${ }^{1}$ Pil-Woo HUH, ${ }^{2}$ Hyung-Seok KIM, ${ }^{1}$ In-Soo KIM, ${ }^{1}$ \\ Seokmin CHOI, ${ }^{1}$ Jin-Ho MOK, ${ }^{1}$ and Choon-Woong $\mathrm{HUH}^{1}$ \\ ${ }^{1}$ Department of Neurosurgery, Myong-Ji St. Mary's Hospital, Seoul, ROK; \\ ${ }^{2}$ Department of Neurosurgery, Uijeongbu St. Mary's Hospital, \\ the Catholic University of Korea College of Medicine, Uijeongbu, ROK
}

\begin{abstract}
Combined superficial temporal artery-middle cerebral artery anastomosis and encephalo-duro-arteriogaleo-synangiosis (EDAGS) were retrospectively compared with indirect bypass, EDAGS with or without inversion, in 134 hemispheres of 96 adult patients with non-hemorrhagic moyamoya disease (MMD) in terms of angiographic findings, perioperative complications, and clinical outcome. Angiographic revascularization seemed to be better in the combined bypass group compared with the EDAGS group ( $p=0.045)$, but perioperative complication tended to be slightly more common in the combined bypass group. No statistical differences were found in clinical outcome. EDAGS is a very reliable alternative to combined bypass in adult MMD. However, randomized clinical trials are needed to assess the long-term efficacy of any bypass surgery in adult patients with MMD.
\end{abstract}

Key words: moyamoya disease, adult, combined direct and indirect bypass surgery, indirect bypass surgery

\section{Introduction}

Adult patients with moyamoya disease (MMD) seem to have a different response to direct or indirect cerebral revascularization procedures compared to children. In particular, the angiographic findings after indirect bypass surgery have been relatively inconsistent in adults, but are very reliable in pediatric patients. ${ }^{14,18,27,31)}$ These differences between adult and pediatric MMD suggest that an age-specific tailored approach is required for the management of patients with MMD.

Direct bypass procedures such as superficial temporal artery-middle cerebral artery (STA-MCA) anastomosis are frequently performed in adult patients with MMD because the donor and recipient vessels have suitable sizes for direct anastomosis in most cases, and this procedure provides immediate augmentation of cerebral blood flow in the ischemic cerebral cortex affected by MMD. However, direct bypass surgery-related various complications such as hyperperfusion, cerebral infarction, intracerebral hemorrhage, or even death

Received December 5, 2011; Accepted January 5, 2012 have been reported. ${ }^{8,10,12,15,16,20,26,35,36)}$ In contrast, indirect bypass surgery procedures, such as encephalo-duro-arterio-synangiosis (EDAS), encephalo-myo-synangiosis (EMS), and encephalo-duroarterio-myo-synangiosis (EDAMS), are considered easier and safer even in patients with medical comorbidity, and slow but steady revascularization can be maintained because the STA, deep temporal artery, or middle meningeal artery (MMA) are little affected by MMD.

The present study compared combined STA-MCA anastomosis and encephalo-duro-arterio-galeosynangiosis (EDAGS) to EDAGS with or without inversion in adult patients with non-hemorrhagic MMD in terms of angiographic findings, perioperative complications, and clinical outcome.

\section{Materials and Methods}

The medical records and results of neuroimaging studies were retrospectively reviewed of 134 bypass surgeries (72 combined bypass and 62 EDAGS) performed in 96 adult patients with MMD, 28 males and 68 females aged 18-68 years (mean 38.44 years), who underwent bypass surgery at our institutions between February 1998 and January 2010. The 134 by- 
pass surgeries included 74 bilateral (38 combined, 24 EDAGS, 12 mixed) and 60 unilateral procedures (28 combined, 32 EDAGS). Clinical presentations included cerebral infarction in 61 patients (combined in 34, EDAGS in 27), transient ischemic attack (TIA)reversible ischemic neurological deficit in 49 patients (combined in 25, EDAGS in 24), TIA-infarction in 10 patients (combined in 4, EDAGS in 6), and other symptoms including headache, dizziness, and involuntary movement in 14 patients. Inclusion criteria for bypass surgery were non-hemorrhagic and symptomatic MMD, no recent cerebral infarction within 6 weeks, decreased cerebrovascular reserve on perfusion study using hexamethylpropyleneamine oxime single photon emission computed tomography or perfusion computed tomography, and Karnofsky performance status score $\geq 70$.

The primary surgical modality was combined STA-MCA anastomosis using the $\mathrm{M}_{3}$ or $\mathrm{M}_{4}$ cortical branch of the MCA and the larger of the 2 branches of the STA, EDAGS using the other branch of the STA with or without inversion, and dural pedicle inversion (DPI) ${ }^{6,19)}$ Inversion of EDAGS ${ }^{19)}$ was used in 30 procedures (20 combined, 10 EDAGS) in 22 patients aged $\geq 50$ years in consideration of poor revascularization of simple EDAS, especially in patients older than 40 years, ${ }^{27)}$ and personal experience (unpublished data). Indirect bypass surgery using EDAGS and DPI was chosen if the size of the donor or recipient artery was unsuitable, contribution of branches of the STA to pre-existing ipsilateral or contralateral transdural collateralization, great difference in size of the branches of the STA, and presence of medical comorbidity.

Postoperative angiographic revascularization was evaluated on the lateral view of selective external carotid angiography and was classified into 3 groups: good, fair, and poor. ${ }^{24)}$ The clinical outcomes were divided into 4 categories: excellent, good, fair, and poor. ${ }^{21)}$ Follow-up duration was 2 to 16 months (mean 6.8 months) for the evaluation of postoperative angiographic findings, 1 day to 6 weeks (mean 4.2 weeks) for early perioperative complications, and 1 day to 108 months (40.2 months) for clinical outcome. Statistical analysis used the unpaired t-test, chi-square test, and Fisher's exact test, and adopted a significance level of 0.05 .

\section{Results}

The postoperative angiographic revascularization findings, perioperative complications, and clinical outcomes are summarized in Table 1 . Good revascularization was found in 57 of 71 sides $(80.3 \%)$ treated by combined bypass and 46 of 61 sides $(75.4 \%)$ treated by indirect bypass. Fair revascularization was obtained in 10 of 71 sides after combined bypass and in 9 of 61 sides after indirect bypass. However, only poor revascularization was achieved in 4 sides with combined bypass and 6 with indirect bypass. The 2 bypass groups showed significant statistical difference in the extent of angiographic collateralization $(p=0.045)$. Perioperative overall neurological complications occurred in 17 of 71 procedures $(23.9 \%)$ in the combined bypass group, which included 5 major complications (modified Rankin scale [mRS] 3-5) and 12 minor complications (mRS 1-2), and in 12 of 61 procedures (19.7\%) in the indirect bypass group, which included 3 major and 9 minor complications. Seven of 8 major neurological complications in both bypass groups were related to ipsilateral $(n=4)$ and contralateral $(n=3)$ cerebral infarction and one intracerebral hemorrhage occurred with combined bypass surgery. Perioperative neurological complications tended to be slightly more common in the combined bypass group, but no significant difference was found between the two bypass groups. Perioperative non-neurological complications such as delayed wound healing or wound infection occurred in 3 sides of the combined bypass group and 2 sides of the indirect bypass group. Clinical outcome was favorable (excellent and good) in 59 of 71 procedures $(83.1 \%)$ in the combined bypass group and in 50 of 61 procedures $(82.0 \%)$ in the indirect bypass group, and unfavorable (fair and poor) in 12 of 71 procedures $(16.9 \%)$ in the combined bypass group and in 11 of 61 procedures $(18.0 \%)$ in the indirect bypass group. Two deaths, one in each group, were related to acute myocardial infarction after bypass surgery, but before postoperative angiographic evaluation. Clinical outcome showed no statistical difference between the two bypass groups.

\section{Discussion}

Extracranial to intracranial bypass surgery has proven a reliable method for the treatment of patients with non-hemorrhagic MMD in terms of clinical outcomes as well as angiographic revascularization. Direct or combined direct and indirect or indirect bypass procedures have been utilized in patients with MMD, especially with ischemic presentation, but the safety and efficacy of these surgical techniques remain largely unproven. Furthermore, the optimal surgical treatment in adult patients with MMD is unknown. ${ }^{33)}$ Direct bypass such as STA-MCA anastomosis ${ }^{15,17,22,24,25)}$ or combination of direct and indirect bypass surgery ${ }^{11,13,27,29)}$ have been recommended in adult patients with 
Table 1 Summary of clinical data of 96 adult patients with moyamoya disease who underwent 134 bypass surgeries

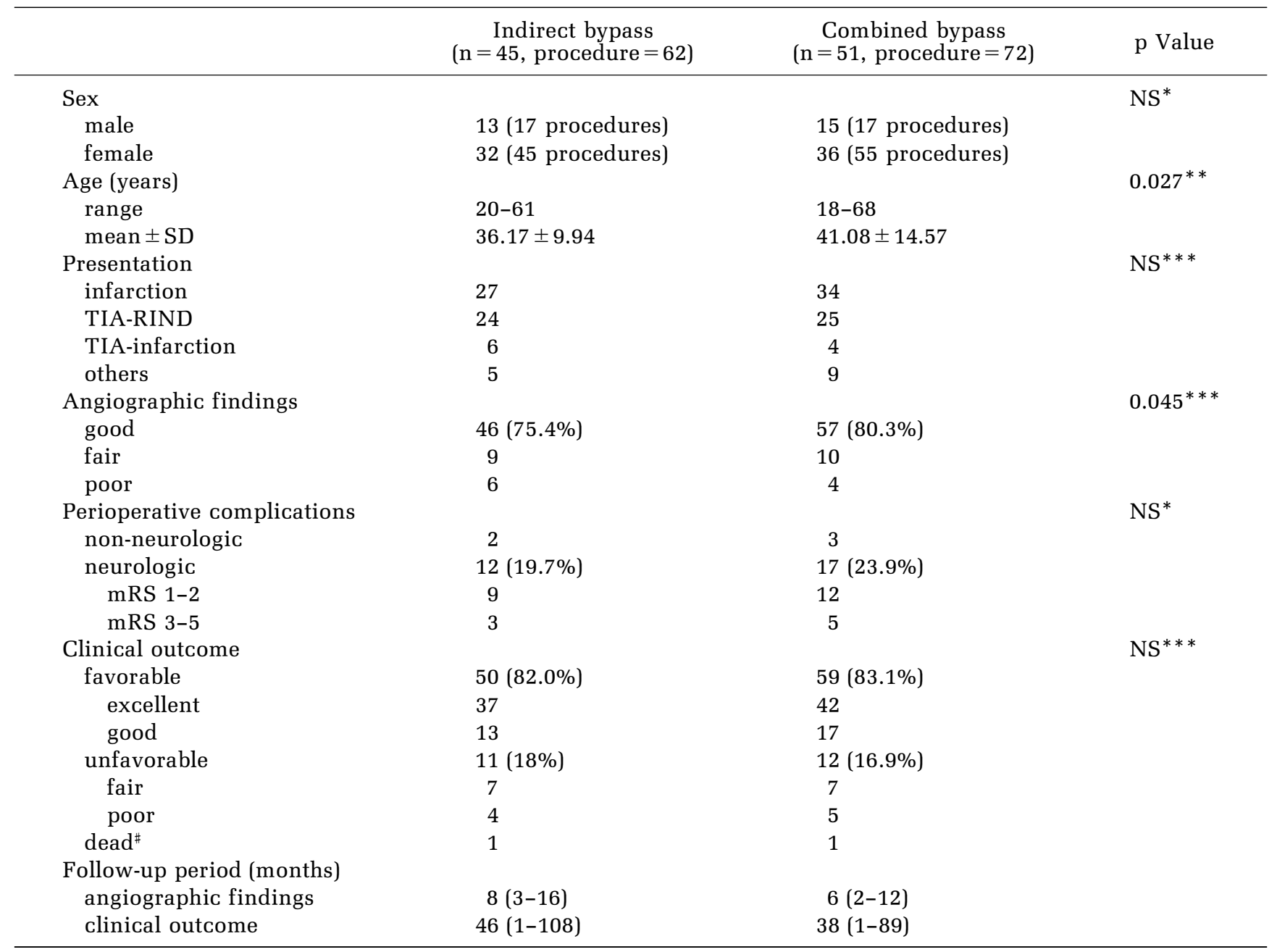

${ }^{*}$ Chi-square test, ${ }^{* *}$ unpaired t-test, ${ }^{* * *}$ Fisher's exact test. \#Two patients died before postoperative angiographic evaluation. mRS: modified Rankin scale, NS: not significant, RIND: reversible ischemic neurologic deficit, SD: standard deviation, TIA: transient ischemic attack.

MMD because these procedures are effective at preventing future ischemic events and improve the quality of life. ${ }^{10)}$ Recent studies have documented real augmentation of collateral blood flow using color or laser Doppler flowmetry, indocyanine green angiography, or perivascular ultrasonic flowprobe investigations. ${ }^{2,3,9,23,37)}$ On the other hand, some complications are related to direct bypass surgery such as symptomatic hyperperfusion and cheirooral syndrome, which are mostly not permanent, cerebral infarction, intracerebral hemorrhage, or even death. $8,10,12,15,16,20,26,32,35,36)$ The long-term durability of direct bypass surgery for cerebral perfusion in MMD has yet to be elucidated because the longterm patency of the recipient artery such as the MCA branches in MMD remains unknown. ${ }^{1,28)}$

In contrast, indirect bypass surgery is generally considered easier and safer, with shorter surgery time, less invasive compared with direct anastomosis, and more feasible in patients with inadequate recipient or donor arteries. ${ }^{5,35)}$ However, indirect bypass requires several weeks to form collateral vessels, ${ }^{15,35)}$ and angiographic revascularization is relatively inconsistent in adult patients compared with pediatric patients. ${ }^{14,18,27,31)}$ Our study showed that combined direct and indirect bypass is slightly superior to EDAGS in the extent of postoperative angiographic revascularization $(p=0.045)$. A recent quantitative angiographic comparison between the direct and indirect revascularization modalities in adult patients with MMD concluded that the extent of revascularization was highest after STA-MCA anastomosis with EDAMS and lowest after only EDAS. ${ }^{4)}$ This study supports our angiographic find- 
ings, although the detailed surgical technique is not the same as our procedure.

Considering the poor revascularization achieved by simple EDAS, especially in patients older than 40 years, ${ }^{27)}$ we have adopted inverted EDAGS and DPI for obtaining abundant and widespread revascularization through the STA and the attached wide galeal tissue by direct contact with the ischemic cerebral cortex in adult patients with MMD 50 years or older. The combined bypass group tended to have slightly higher rates of surgery-related neurological complications compared to the indirect bypass group, especially for major complications. The hemodynamic changes in the STA after direct bypass surgery mainly occurred in the early period after the operation and seems to be related to low resistance or lower perfusion pressure of the intracranial vascular bed, or the large pressure gradient between the donor and recipient vessels.,23,37) Only EDAS promoted adequate pial collateral development and increased perfusion in the majority of North American adult patients with MMD with low rates of postoperative TIAs, infarction, and hemorrhage. ${ }^{34)}$ However, the present study found no significant difference in overall clinical outcome between the groups. Therefore, indirect bypass such as EDAGS seems to be a reasonable approach considering the surgery-related early postoperative complications and overall clinical outcome in our study.

Indirect revascularization by EDAS and burr holes for MMD resulted in long-term resolution of ischemic and hemorrhagic manifestations in $95 \%$ of adults $(\mathrm{n}=33)$ and children $(\mathrm{n}=9)$ with emphasis on the significant contribution of the MMA in angiographic revascularization. ${ }^{7)}$ Our personal experience regarding the main contributor to postoperative revascularization in MMD indicates that the contribution of the dural arteries including the MMA was not inferior to the STA in both the EDAGS group and the combined bypass group (unpublished data). Dominance of EMS compared with the STA was found in the combined group as well as the indirect (EMS) group in eventual patterns of revascularization based on dominancy of donor arteries in MMD. ${ }^{30}$ ) These factors including dominancy of the dural arteries in revascularization and slightly fewer major perioperative complications in the indirect bypass group may have contributed to the absence of significant difference in long-term clinical outcome compared with the combined bypass group.

This study has some limitations. First, this is a non-randomized, controlled study with different indications for combined bypass surgery and indirect bypass surgery, and application of EDAGS and in- verted EDAGS according to the patient's age. The second limitation is inherent to a retrospective study from medical records, which carries the risk of selection bias in the data collection.

The present study suggests that EDAGS with or without inversion and DPI is a very reliable alternative to combined STA-MCA anastomosis and indirect bypass surgery because the clinical outcome and surgery-related complication rates are not significantly different, although combined bypass surgery is slightly superior to indirect bypass surgery in terms of postoperative angiographic revascularization. However, randomized clinical trials are needed to assess the safety and long-term efficacy of bypass surgery in adult patients with MMD.

\section{Acknowledgments}

The authors thanks nurse specialists for stroke, Young-Hee Kang, RN and Ju-Hee Lee, RN, and the intensive care unit staff nurses for their assistance with this study.

\section{References}

1) Asofora WT, West M, McClarty B: Angiography of encephalomyosynangiosis and superficial temporal artery to middle cerebral artery anastomosis in moyamoya disease. AJNR Am J Neuroradiol 14: 29-30, 1993

2) Awano T, Sakatani K, Yokose N, Hoshino T, Fujiwara N, Nakamura S, Murata Y, Kano T, Katayama Y, Shikayama T, Miwa M: EC-IC bypass function in Moyamoya disease and non-Moyamoya ischemic stroke evaluated by intraoperative indocyanine green fluorescence angiography. Adv Exp Med Biol 662: 519-524, 2010

3) Awano T, Sakatani K, Yokose N, Kondo Y, Igarashi T, Hoshino T, Nakamura S, FujiWara N, Murata Y, Katayama Y, Shikayama T, Miwa M: Intraoperative EC-IC bypass blood flow assessment with indocyanine green angiography in moyamoya and nonmoyamoya ischemic stroke. World Neurosurg 73: 668-741, 2011

4) Bang JS, Kwon OK, Kim JE, Kang HS, Park H, Cho SY, Oh CW: Quantitative angiographic comparison with the OSIRIS program between the direct and indirect revascularization modalities in adult moyamoya disease. Neurosurgery 70: 625-633, 2012

5) Chiu D, Shedden P, Bratina P, Grotta JC: Clinical features of moyamoya disease in the United States. Stroke 29: 1347-1351, 1998

6) Dauser RC, Tuite GF, Mccluggage CW: Dural inversion procedure for moyamoya disease. J Neurosurg 86: 719-723,1997

7) Dusick JR, Gonzalez NR, Martin NA: Clinical and angiographic outcomes from indirect revascularization 
surgery for Moyamoya disease in adults and children: a review of 63 procedures. Neurosurgery 68: 34-43, 2011

8) Fujimura M, Mugikura S, Kaneta $T$, Shimizu $H$, Tominaga T: Incidence and risk factors for symptomatic cerebral hyperperfusion after superficial temporal artery-middle cerebral artery anastomosis in patients with moyamoya disease. Surg Neurol 871: 442-447, 2009

9) Gesang DZ, Zhang D, Zhao JZ, Wang S, Zhao YL, Wang R, Sun JJ, Meng Z: Laser Doppler flowmeter study on regional cerebral blood flow on early stage after standard superficial temporal artery-middle cerebral artery bypass surgery for moyamoya disease. Chin Med J (Engl) 122: 2412-2418, 2009

10) Guzman R, Lee M, Achrol A, Bell-Stephens T, Kelly M, Do HM, Marks MP, Steinberg GK: Clinical outcome after 450 revascularization procedures for moyamoya disease. Clinical article. J Neurosurg 111: 927-935, 2009

11) Hallemeier CL, Rich KM, Grubb RL Jr, Chicoine MR, Moran CJ, Cross DT III, Zipfel GJ, Dacey RG Jr, Derdeyn CP: Clinical features and outcome in North American adults with moyamoya phenomenon. Stroke 37: 1490-1496, 2006

12) Hayashi $T$, Shiran R, Fujimura M, Tominaga $T$ : Postoperative neurological deterioration in pediatric moyamoya disease: watershed shift and hyperperfusion. J Neurosurg Pediatr 6: 73-81, 2010

13) Houkin K, Ishikawa T, Yoshimoto $T$, Abe H: Direct and indirect revascularization for moyamoya disease surgical techniques and peri-operative complications. Clin Neurol Neurosurg 99 Suppl 2: S142-S145, 1997

14) Houkin K, Kuroda S, Ishikawa T, Abe H: Neovascularization (angiogenesis) after revascularization in moyamoya disease. Which technique is most useful for moyamoya disease? Acta Neurochir (Wien) 142: 269-276, 2000

15) Ishikawa T, Houkin K, Kamiyama H, Abe H: Effects of surgical revascularization on outcome of patients with pediatric moyamoya disease. Stroke 28: 1170-1173, 1997

16) Iwama T, Hashimoto N, Tsukahara T, Murai B: Perioperative complications in adult moyamoya disease. Acta Neurochir (Wien) 132: 26-31, 1995

17) Karasawa J, Touho H, Ohnishi H, Miyamoto S, Kikuchi $\mathrm{H}$ : Long-term follow-up study after extracranial-intracranial bypass surgery for anterior circulation ischemia in childhood moyamoya disease. J Neurosurg 77: 84-89, 1992

18) Kawamura $T$, Imaizumi $S$, Sakurai $Y$, Uenohara $H$, Nishino A, Suzuki S: [Effect of various surgical procedures for moyamoya disease in children and adults]. No Shinkei Geka 22: 933-938, 1994 (Japanese)

19) Kim DS, Yoo DS, Huh PW, Kang SG, Cho KS, Kim MC: Combined direct anastomosis and encephaloduroarteriogaleosynangiosis using inverted superficial temporal artery-galeal flap and superficial temporal artery-galeal pedicle in adult moyamoya disease. Surg Neurol 66: 389-395, 2006

20) Kim JE, Oh CW, Kwon OK, Park SQ, Kim SE, Kim YK: Transient hyperperfusion after superficial temporal artery/middle cerebral artery bypass surgery as a possible cause of postoperative transient neurological deterioration. Cerebrovasc Dis 25: 580-586, 2008

21) Kim SK, Wang KC, Kim IO, Lee DS, Cho BK: Combined encephaloduroarterio-synangiosis and bifrontal encephalogaleo(periosteal)synangiosis and pediatric moyamoya disease. Neurosurgery 50: 88-96, 2002

22) Krayenbuhl HA: The moyamoya syndrome and the neurosurgeon. Surg Neurol 4: 353-360, 1975

23) Lee M, Guzman R, Bell-Stephens T, Steinberg GK: Intraoperative blood flow analysis of direct revascularization procedures in patients with moyamoya disease. J Cereb Blood Flow Metab 31: 262-274, 2011

24) Matsushima T, Inoue T, Suzuki SO, Fujii K, Fukui M, Hasuo K: Surgical treatment of moyamoya disease in pediatric patients-comparison between the results of indirect and direct revascularization procedures. Neurosurgery 31: 401-405, 1992

25) Matsushima Y, Aoyagi M, Koumo Y, Takasato $Y$, Yamaguchi T, Masaoka H, Suzuki R, Ohno K: Effects of encephalo-duro-arterio-synangiosis on childhood moyamoya patients-swift disappearance of ischemic attacks and maintenance of mental capacity. Neurol Med Chir (Tokyo) 31: 708-714, 1991

26) Mesiwala AH, Sviri G, Fatemi N, Britz GW, Newell DW: Long-term outcome of superficial temporal artery-middle cerebral artery bypass for patients with moyamoya disease in the US. Neurosurg Focus 24(2): E15, 2008

27) Mizoi K, Kayama T, Yoshimoto T, Nagamine Y: Indirect revascularization for moyamoya disease: is there a beneficial effect for adult patients? Surg Neurol 45: 541-549, 1996

28) Nakagawa $Y$, Abe $H$, Sawamura $Y$, Kamiyama $H$, Gotoh S, Kashiwaba T: Revascularization surgery for moyamoya disease. Neurol Res 10: 32-39, 1988

29) Nakashima H, Meguro T, Kawada S, Hirotsune N, Ohmoto T: Long-term results of surgically treated moyamoya disease. Clin Neurol Neurosurg 99 Suppl 2: S156-S161, 1997

30) Saito $N$, Imai $H$ : Insights on the revascularization mechanism for treatment of Moyamoya disease based on the histopathologic concept of angiogenesis and arteriogenesis. World Neurosurg 75: 204-205, 2011

31) Sakamoto S, Ohba S, Shibukawa M, Kiura Y, Okazaki T, Equchi K, Sugiyama K, Kurisu K: Angiographic neovascularization after bypass surgery in moyamoya disease: our experience at Hiroshima University Hospital. Hiroshima J Med Sci 56: 29-32, 2007

32) Sasamori T, Kuroda S, Nakayama N, Iwasaki Y: Incidence and pathogenesis of transient cheiro-oral syndrome after surgical revascularization for moyamoya disease. Neurosurgery 67: 1054-1060, 2010 
33) Starke RM, Komotar RJ, Connolly ES: Optimal surgical treatment for moyamoya disease in adults: direct versus indirect bypass. Neurosurg Focus 26(4): E8, 2009

34) Starke RM, Komotar RJ, Hickman ZL, Paz YE, Pugliese AG, Otten ML, Garrett MC, Elkind MS, Marshall RS, Festa JR, Meyers PM, Connolly ES Jr: Clinical features, surgical treatment, and long-term outcome in adult patients with moyamoya disease. Clinical article. J Neurosurg 111: 936-942, 2009

35) Ueki K, Meyer FB, Mellinger JF: Moyamoya disease: the disorder and surgical treatment. Mayo Clin Proc 69: 749-757, 1994

36) Uno M, Nakajima N, Nishi K, Shinno K, Nagahiro S: Hyperfusion syndrome after extracranial-intracranial bypass in a patient with moyamoya disease—case report. Neurol Med Chir (Tokyo) 38: 420-424, 1998

37) Wu M, Huang Z, Zhang D, Wang L, Sun J, Wang S, Zhao Y, Zhao J: Color doppler hemodynamic study of the superficial temporal arteries in superficial temporal artery-middle cerebral artery (STA-MCA) bypass surgery for Moyamoya disease. World Neurosurg 75: 258-263, 2011

Address reprint requests to: Pil-Woo Huh, MD, Department of Neurosurgery, Uijeongbu St. Mary's Hospital, The Catholic University of Korea College of Medicine, 65-1, Kumoh-dong, Uijeongbu 480-717, Korea. e-mail: dalskim@catholic.ac.kr 